\title{
Six3 repression of Wnt signaling in the anterior neuroectoderm is essential for vertebrate forebrain development
}

\author{
Oleg V. Lagutin, ${ }^{1}$ Changqi C. Zhu, ${ }^{1}$ Daisuke Kobayashi, ${ }^{2}$ Jacek Topczewski, $^{3}$ Kenji Shimamura, $^{2}$ \\ Luis Puelles, ${ }^{4}$ Helen R.C. Russell, ${ }^{1}$ Peter J. McKinnon, ${ }^{1}$ Lilianna Solnica-Krezel, ${ }^{3}$ \\ and Guillermo Oliver ${ }^{1,5}$

\begin{abstract}
${ }^{1}$ Department of Genetics, St. Jude Children's Research Hospital, Memphis, Tennessee 38105-2794, USA; ${ }^{2}$ Department of Neurobiology, Graduate School of Medicine, University of Tokyo, Bunkyo-ku, Tokyo 113-0033, Japan; ${ }^{3}$ Department of Biological Sciences, Vanderbilt University, Nashville, Tennessee 37232, USA; ${ }^{4}$ Department of Morphological Sciences, Faculty of Medicine, University of Murcia, E-30100 Murcia, Spain
\end{abstract}

In vertebrate embryos, formation of anterior neural structures requires suppression of Wnt signals emanating from the paraxial mesoderm and midbrain territory. In $\mathrm{Six}^{-/-}$mice, the prosencephalon was severely truncated, and the expression of Wnt1 was rostrally expanded, a finding that indicates that the mutant head was posteriorized. Ectopic expression of Six 3 in chick and fish embryos, together with the use of in vivo and in vitro DNA-binding assays, allowed us to determine that Six3 is a direct negative regulator of Wnt1 expression. These results, together with those of phenotypic rescue of headless/tcf3 zebrafish mutants by mouse Six 3 , demonstrate that regionalization of the vertebrate forebrain involves repression of Wnt1 expression by Six3 within the anterior neuroectoderm. Furthermore, these results support the hypothesis that a Wnt signal gradient specifies posterior fates in the anterior neural plate.

[Keywords: Six3; forebrain; mouse; homeobox; Wnt; zebrafish]

Received November 15, 2002; revised version accepted December 9, 2002.

Nieuwkoop's two-signal model proposed that induced neural tissue is inherently anterior (forebrain) in character and that a graded transforming (or posteriorizing) signal specifies posterior identity to the anterior neuroectoderm (Nieuwkoop 1952). It has been suggested that during vertebrate head development, the level of Wnt activity may specify posterior-to-anterior fates within the neural plate (Niehrs 1999; Heisenberg et al. 2001; Kiecker and Niehrs 2001). Wnt signaling must be inhibited to allow the development of the rostral telencephalon, or the prospective forebrain will acquire a caudal diencephalic identity (Niehrs 1999; Heisenberg et al. 2001; Kiecker and Niehrs 2001). This anterior Wnt-signaling-free zone is maintained by Wnt antagonists secreted by the anterior neuroectoderm and adjacent anterior mesendoderm (Niehrs 1999; Houart et al. 2002).

Head truncations occur when genes that are required for the development of the anterior visceral endoderm (AVE; i.e., Hex, Lim1, and Otx2) are mutated (Thomas and Beddington 1996; Shawlot et al. 1999; Martinez-Bar-

${ }^{5}$ Corresponding author.

E-MAIL guillermo.oliver@stjude.org; FAX (901) 526-2907.

Article published online ahead of print. Article and publication date are at http://www.genesdev.org/cgi/doi/10.1101/gad.1059403. bera and Beddington 2001; Perea-Gomez et al. 2001). The lack of anterior head structures also occurs in mice that are double-homozygous for chordin and noggin, which encode secreted bone morphogenetic protein antagonists (Bachiller et al. 2000). In addition, mouse embryos lacking Dickkopf1 (Dkk1), a secreted protein that acts as an inhibitor of the Wnt coreceptor LRP-6, lack head structures anterior to the midbrain; $D k k 1$ activity is required in the axial mesendoderm (Mukhopadhyay et al. 2001). Variable forebrain truncations are also observed in mice with inactivating mutations in the homeobox gene Hesx1, whose activity is required in the anterior neural ectoderm (Martinez-Barbera and Beddington 2001).

We have previously shown that in mice, Six3 is expressed in the most anterior part of the developing neural plate (Oliver et al. 1995). To determine the role of Six3 during vertebrate development, we inactivated the mouse Six3 locus. We find that Six3 is required for development of the mammalian rostral forebrain. The absence of Six 3 results in forebrain truncations and posteriorization of the remaining mutant head. We demonstrate that Six 3 binds to the Wnt1 promoter region in vivo and represses Wnt 1 expression in the most anterior neuroectoderm. Work recently performed in zebrafish embryos has suggested that telencephalic induction, as 
well as the subsequent patterning of the forebrain into telencephalic, eye, and diencephalic regions, is the result of the graded expression of Wnt signaling in the anterior neural plate (Houart et al. 2002).

Thus, during vertebrate head regional specification, the maintenance and refinement of anterior neural fates requires that Wnt signaling is transcriptionally repressed in the anterior neuroectoderm, and Six3 is a key player during this process. We also show that Six 3 is sufficient to suppress the loss of forebrain resulting from excess Wnt1 signaling in headless (Tlc3) zebrafish mutants. Taken together, these results not only identified Six3 as a key player in vertebrate head development, but also demonstrated the existence of another regulatory step in the complex Wnt signaling pathway, the direct repression of Wnt1 expression by a transcription factor in the mammalian anterior neural plate at the late headfoldearly somite stage, a step that is probably required for the maintenance of the anterior neural fates.

\section{Results}

Six3 is required for the development of the forebrain

To determine the role of Six3 during vertebrate development, in the present study the mouse Six3 locus was inactivated by an in-frame insertion of $1 a c Z$, the gene that encodes $\beta$-galactosidase ( $\beta$-gal; Fig. 1$)$. The resulting $\beta$-gal activity allowed analysis of Six3 expression throughout development. Although a form of human holoprosencephaly is caused by mutations in the Six3 homeodomain (Wallis et al. 1999) or Six domain (Pasquier et al. 2000), Six3-heterozygous mice exhibited an apparently normal morphologic appearance (Fig. 2E; data not shown). Six3-null embryos died at birth (total absence of Six3 expression was determined by immunofluorescence with a specific anti-Six3 antibody; data not shown); the embryos lacked most head structures anterior to the midbrain, including the eyes and nose (Fig. 2B,D,F). The rest of the body axis appeared normal. Skeletal prepara-
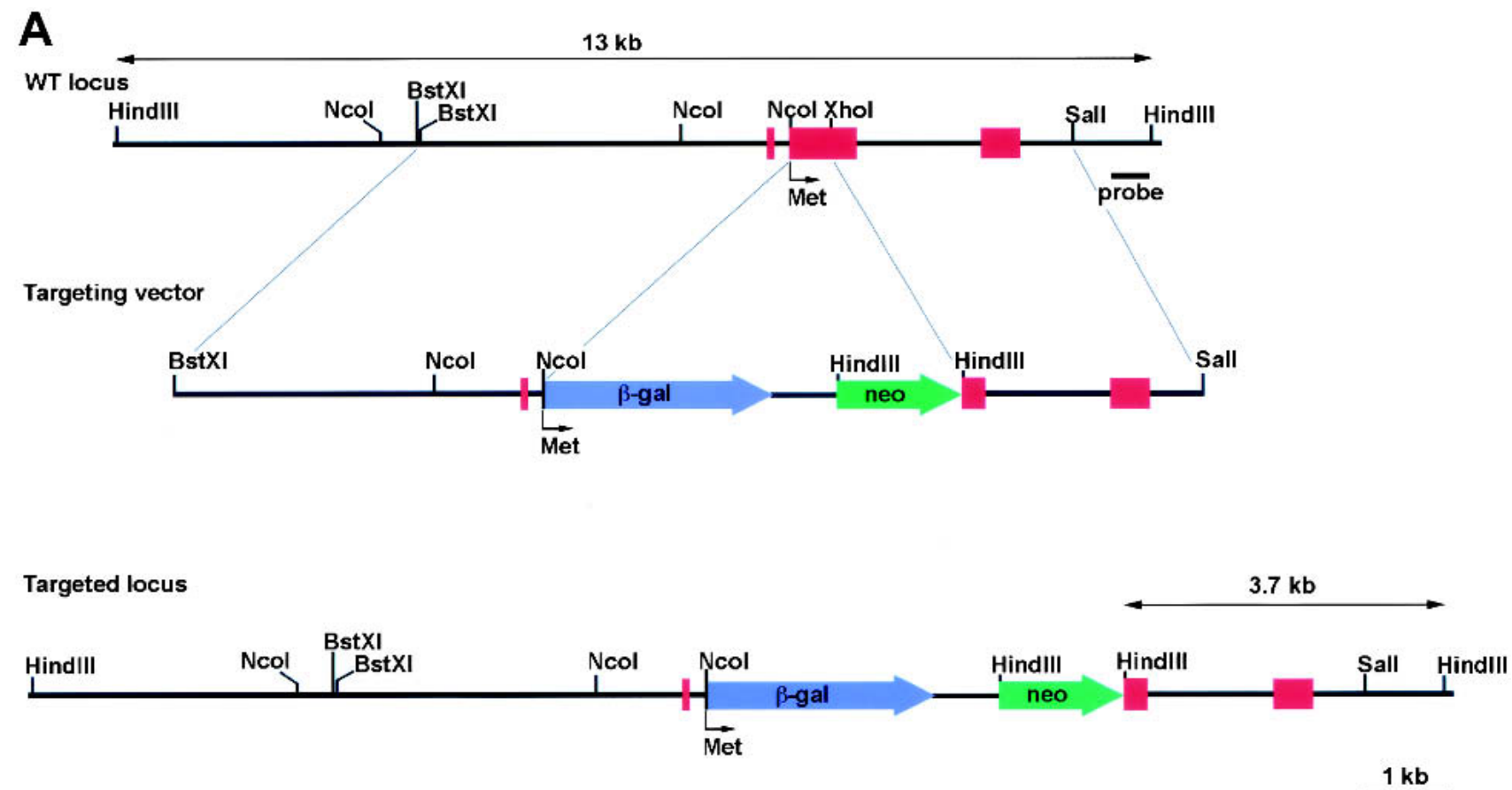

\section{B}

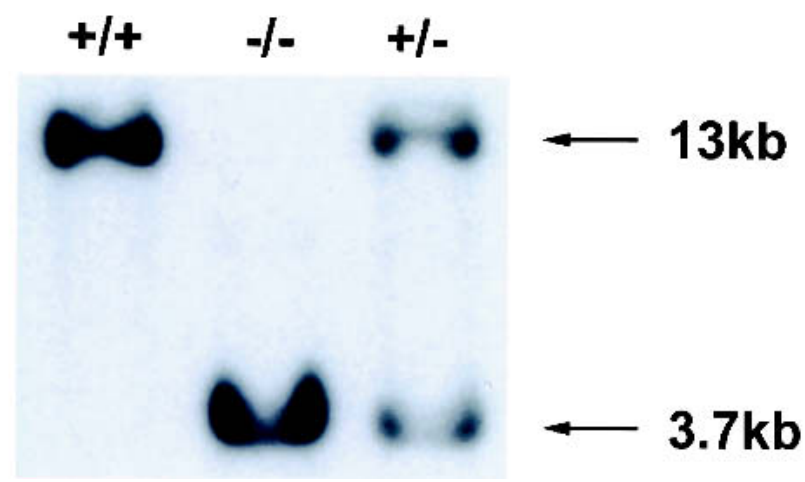

Figure 1. Targeted disruption of Six3. (A) Six3 was inactivated by the in-frame insertion of a $\beta$-galactosidase-neomycin resistance cassette in the NcoI-XhoI site located 22 amino acids downstream of the first initiation methionine. (B) In embryonic stem cells, the targeted Six3 locus was identified by Southern blot analysis of genomic DNA digested with HindIII and hybridized to a $0.5-\mathrm{kb}$ external genomic fragment. The $13.0-\mathrm{kb}$ fragment originated from the wild-type allele, and the $\sim 3.7-\mathrm{kb}$ fragment originated from the targeted allele. 
Lagutin et al.

Figure 2. Inactivation of Six3 results in the absence of the eyes and nose and leads to postnatal lethality. Wild-type $(A, C)$, $\operatorname{Six}^{+/-}(E)$, and Six3 ${ }^{-/-}(B, D, F)$ embryos were used. Staining of bone (red) and cartilage (blue) of wild-type $(C)$ and Six3-null $(D)$ embryos revealed substantial stunting of the rostral skull (arrow) and maxillofacial derivatives; the mandible is also indicated (arrowhead). (E) In an E12.5 Six3-heterozygous embryo, X-gal staining recapitulated the normal pattern of Six3 expression in the eyes, nose (black arrowhead), midbrain, pretectal tegmentum (the white arrow is placed at the boundary between midbrain and pretectum, just caudal to the posterior commissure), ZLI (white arrowhead), and rostral ventral thalamus (red arrow). (F) In the $\mathrm{Six}^{-/-}$littermates, the residual X-gal staining was detected throughout the midbrain and forebrain tegmentum, the pretectum (weakly stained), and in transverse bands, which may correlate with the ZLI (white arrowhead) and rostral part of the ventral thalamus (red arrow).
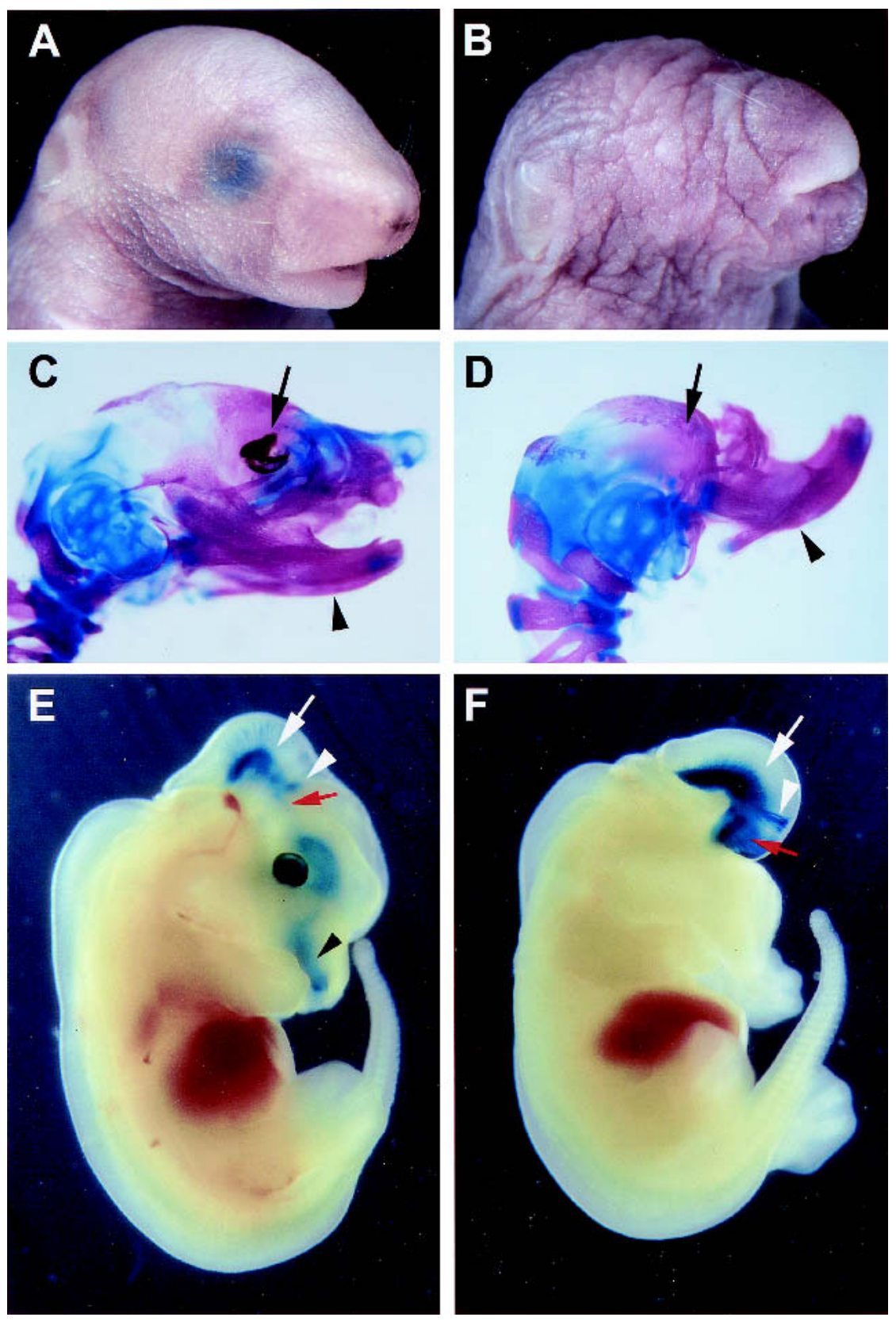

tions of wild-type and Six3-null newborns revealed that the entire rostral skull and the maxillofacial derivatives of the mutant mice were stunted (Fig. 2C,D). Staining of embryonic day 12.5 (E12.5) Six3-heterozygous and Six3null embryos for $\beta$-gal activity revealed severe truncations of the mutant forebrain, including the absence of the telencephalic and optic vesicles (Fig. 2E,F). These results show that Six3 activity is required for forebrain development in vertebrates.

Using a variety of specific molecular markers, we confirmed the absence of the telencephalon and the apparent overall reduction of the forebrain of E9.5 Six3-null embryos (Fig. 3). Mutant embryos lacked telencephalic vesicles, eyes, and olfactory placodes. In wild-type embryos, the expression of Bf1 (Tao and Lai 1992) marked the telencephalic neuroepithelium (Fig. 3A); Bf1 expression was not detected in Six3 ${ }^{-/-}$littermates (Fig. 3B). Furthermore, the homeobox gene Emx2 (Simeone et al. 1992), which is normally expressed in the dorsal forebrain, was not expressed in mutant embryos (Fig. 3C,D). $F g f 8$, a gene normally expressed in the commissural plate and isthmus (Fig. 3E; Crossley and Martin 1995; Shimamura and Rubenstein 1997), was not detected in the area corresponding to the commissural plate but was expressed normally in the isthmus of $\mathrm{Six}^{-/-}$embryos (Fig. 3F).

To investigate dorso-ventral patterning in Six3-null mutants, we analyzed the expression of Pax6 (an alar plate marker) and that of $N k x 2.1$ and $S h h$ (basal/floorplate markers) in E9.5 $\mathrm{Six3}^{-/-}$embryos. The expression 

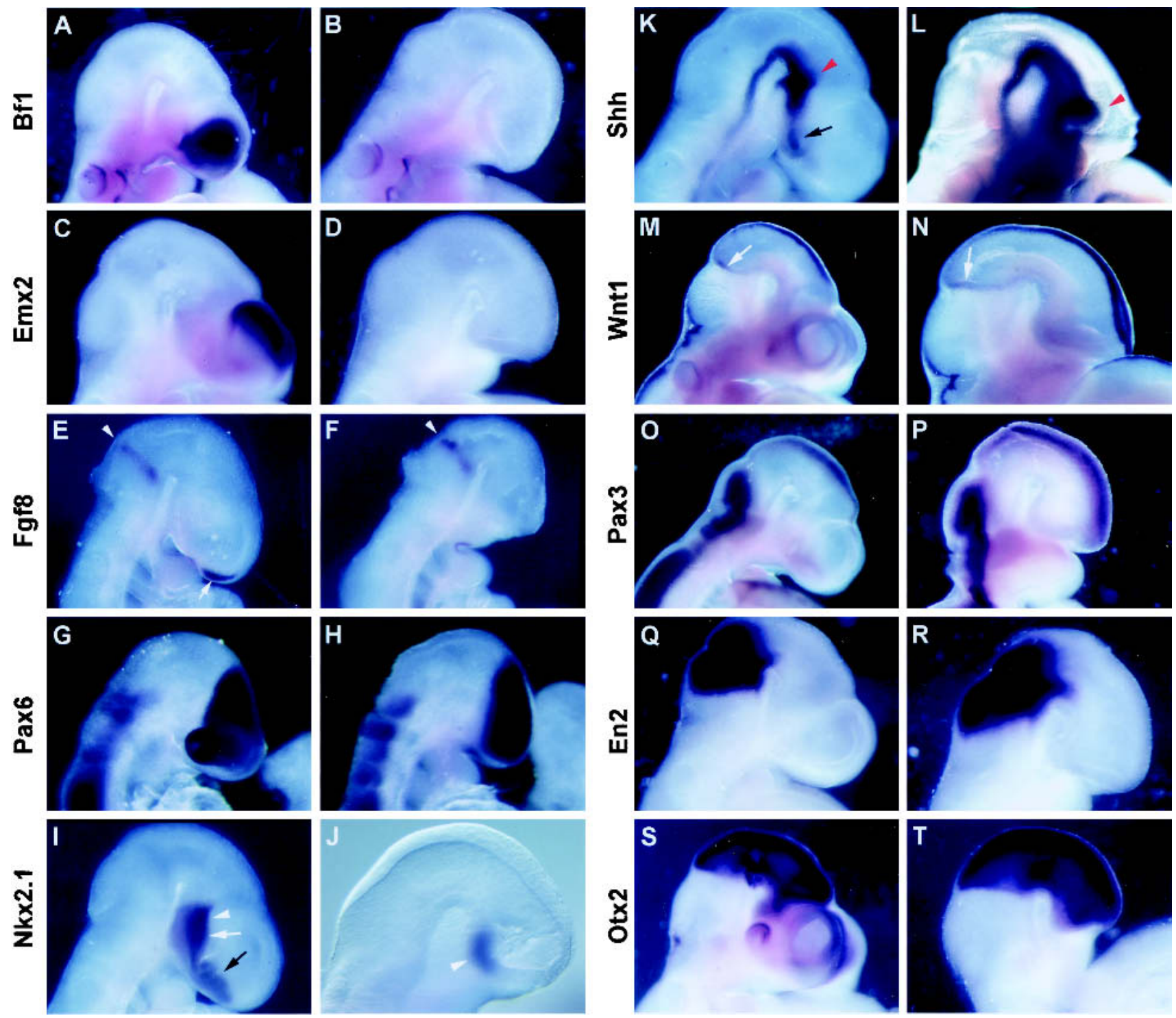

Figure 3. Six3 ${ }^{-/-}$embryos lack a rostral forebrain. Whole-mount in situ hybridization of E9.5-E10.0 wild-type and Six3-null embryos. $B f 1(A)$ and $E m x 2(C)$ were expressed in the telencephalic vesicles of the wild-type embryos but not in the Six $3^{-/-}$littermates $(B, D)$ Fgf8 was expressed in the commissural plate (arrow) and isthmus (arrowheads) of wild-type embryos $(E)$ but only in the isthmus (arrowhead) of $\operatorname{Six}^{-/-}$embryos $(F)$. In wild-type embryos $(G)$, Pax6 expression was localized in the telencephalon and alar diencephalon, including the optic vesicles; in the Six $3^{-/-}$embryos $(H)$, Pax6 expression was normal, except in the missing telencephalic vesicles and eyes. (I) In wild-type embryos, Nkx2.1 was expressed in the posterior hypothalamus (arrowhead), infundibular hypothalamus (white arrow), and basal telencephalon (black arrow). (J) In the Six3-null littermates, only the most caudal portion of the wild-type Nkx2.1 ventral expression domain was present in the posterior hypothalamus. $(K)$ At E10.0, Shh expression extended along the ventral diencephalon into the postchiasmatic forebrain (black arrow) and the ZLI (red arrowhead) of wild-type embryos. $(L)$ The rostral area of

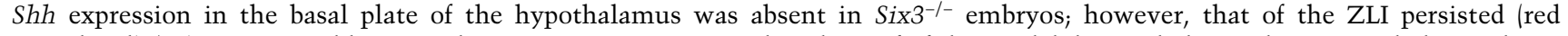
arrowhead). (M) In E10.0 wild-type embryos, Wnt1 was expressed in the roof of the caudal diencephalon and mesencephalon and in a transverse band in the isthmus (arrow). (N) In Six3-null embryos, the Wnt1 expression in the diencephalic roof was rostrally expanded, but that in the isthmus appeared normal. Compared with the expression of the midbrain marker Pax3 in E10.0 wild-type embryos (O), that in the truncated Six3-null forebrain was expanded rostrally in the dorsal alar plate (P). (Q) En2 was expressed across the midbrain-hindbrain boundary in E10.0 wild-type embryos. $(R)$ Although apparently expanded, the pattern of En2 expression was maintained in E10.0 Six3-null littermates. (S) In wild-type E10.0 embryos, Otx2 was expressed in the forebrain and midbrain. (T) In Six3-null littermates, Otx2 expression extended to the anterior end of the mutant forebrain.

of Pax6 (Walther and Gruss 1991) in the alar plate extended to the anterior end of the truncated forebrain (Fig. 3G,H). Nkx2.1 (Lazzaro et al. 1991) expression was absent in the floor of the truncated forebrain and the in- fundibular hypothalamus but was detected rostral to the zona limitans intrathalamica (ZLI) in a small area of the basal plate that resembles the posterior hypothalamus (Fig. 3I,J). In E10.0 Six3 ${ }^{-/-}$embryos, the pattern of Shh 
expression in the forebrain basal plate was reduced in length but extended into the rostral end of the truncated forebrain, where it overlapped with the residual domain of $N k x 2.1$ expression (Fig. 3K,L). At this stage, Shh expression was detectable in the ZLI of the Six3 ${ }^{-/-}$embryos (Fig. 3L). Expression of $R x$, a marker of the optic vesicles and ventral forebrain (Mathers et al. 1997), was not detected in the Six3-null embryos (data not shown). Together, these data indicate that removal of Six3 functional activity results in severe forebrain truncations anterior to the ZLI.

Surgical removal of the rostral portion of the anterior midline tissue of the mouse embryo causes forebrain truncations and rostral expansion of Wnt1 expression (Camus et al. 2000). To determine whether the lack of rostral forebrain in Six3-null embryos leads to the anterior extension of genes normally expressed within the mesencephalon or caudal diencephalon, we examined the expression of midbrain markers in the Six3-null embryos. Wnt1 expression in the roof plate of the midbrain and isthmus appeared normal; however, Wnt1 expression in the diencephalon had clearly extended rostrally into the entire anterior region of the mutant forebrain (rostral to the ZLI; (Figs. 3M,N, 5E, below). Pax3, an alar plate marker in the midbrain and caudal diencephalon, extended into the rostral portion of the mutant forebrain (Fig. 3O,P). Therefore, the expression of at least two dorsal markers of the caudal diencephalon extended anteriorly in the Six3-null embryos, thereby modifying the molecular specification of the presumptive dorsal and ventral thalami on both sides of the ZLI. We also analyzed the expression of En2 (Davis and Joyner 1988) and Otx2 (Simeone et al. 1993). In E10.0 Six3-null embryos, the pattern of En2 expression appeared to be expanded rostrally (Fig. 3Q,R); little or no expression of Otx2 is generally detected in the diencephalon rostral to the ventral thalamus (Fig. 3S). In Six3-null mice, the expression of Otx2 extended anteriorly from the midbrain-hindbrain boundary to the whole anterior region of the truncated forebrain (Fig. 3T). Bromodeoxyuridine incorporation and TUNEL assays performed in E7.5-E9.5 mutant embryos revealed that the forebrain truncation was not caused by altered rates of cell proliferation or cell death (data not shown). Taken together, these results demonstrated that in mice, lack of Six3 function leads to the partial caudalization of the mutant head.

\section{Six3-null head is posteriorized}

Six3 is not expressed in the mouse AVE or prechordal mesoderm; Six3 expression is first detected at $\sim$ E7.0 E7.5 in the anterior neuroectoderm (Lagutin et al. 2001), which at E8.0-E8.5 includes the anterior neural ridge (ANR) and eye field. No obvious alterations in the expression of Hesx1 (Fig. 4A,B) or Dkk1 (data not shown) were observed in the AVE of E7.0-E7.5 Six3-null embryos. A few hours later (E7.5-E8.0), apparently normal Hesx 1 expression was also detected in the anterior neuroectoderm adjacent to the AVE (Fig. 4C,D). The first indication that anterior patterning is affected in Six3- null embryos became apparent at around the 1-2-somite stage. Although Hesx 1 expression remains robust at this stage, it started to diminish in the lateral borders of the anterior neuroectoderm (Fig. 4E,F). This result suggested that the early steps leading to anterior neural induction are unaffected in Six3-null embryos.

Scanning electron micrographs of E8.0-E8.25 wildtype (Fig. 4G) and Six3-null (Fig. 4H) embryos indicated that forebrain morphogenesis is already affected at this early (5-8) somite stage. At this stage, the optic pits were obvious in the wild-type embryo (Fig. 4G) but were not detectable in the mutant littermates (Fig. 4H). In addition, typical thickening in the region corresponding to the ANR in the anterior neuroectodermal border was absent in the Six3-null embryos at this stage, and the midbrain region appeared to be anteriorly expanded (Fig. 4H).

We next compared the expression of markers whose function is required in the anterior neural plate during forebrain development. In contrast to earlier stages, at the 4-6-somite stage, Hesx1 (Martinez-Barbera et al. 2000; Martinez-Barbera and Beddington 2001) expression persisted in Six3-null embryos but only at a very reduced level in a smaller medial domain of the anterior neural plate (Fig. 4I,J). In E8.5 wild-type embryos, $R x$ is expressed in the anterior neural plate, including the retinal field area (Fig. 4K; Mathers et al. 1997). An almost undetectable level of $R x$ expression was observed toward the medial aspect of the anterior neural plate in the Six3null littermates (Fig. 4L). Fgf8 was expressed in the ANR and midbrain-hindbrain isthmus of wild-type embryos (Fig. 4M) but was not detected in the anterior neuroectoderm of Six3 ${ }^{-/-}$littermates; however, Fgf8 expression in the isthmus of the Six3-null embryos was unaffected (Fig. 4N, arrowhead). Bf1 expression, which is normally first detected in the non-neural ectoderm neighboring the anterior neural plate and later in the anterior neuroectoderm (Fig. 4O), was only occasionally detected at a low level in the non-neural ectoderm of Six3-null littermates (Fig. 4P). At this early (3-5) somite stage, Pax3 expression already delineated the future midbrain region in wild-type embryos (Fig. 4Q). In Six3-null littermates, Pax3 expression extended anteriorly (Fig. 4R), thereby corroborating the observed rostral expansion of the midbrain-caudal diencephalic region. These results indicate that anterior neural induction is normal in Six3-null embryos; however, the subsequent steps leading to rostral forebrain formation are arrested.

\section{Six3 is a direct in vivo repressor of Wnt1 expression in the anterior neuroectoderm}

Analysis of Wnt1 expression at the 1-2-somite stage revealed that its level of expression in the midbrain depended on the level of Six3 activity in the forebrain. The level of Wnt1 expression was barely detectable at $~ \mathrm{E} 8.0$ in wild-type embryos (Fig. 5A). In the future midbrain region of Six3-heterozygous littermates, only sparse Wnt1 expression was detected (Fig. 5B), but high levels were consistently seen in this region in the Six3-null 

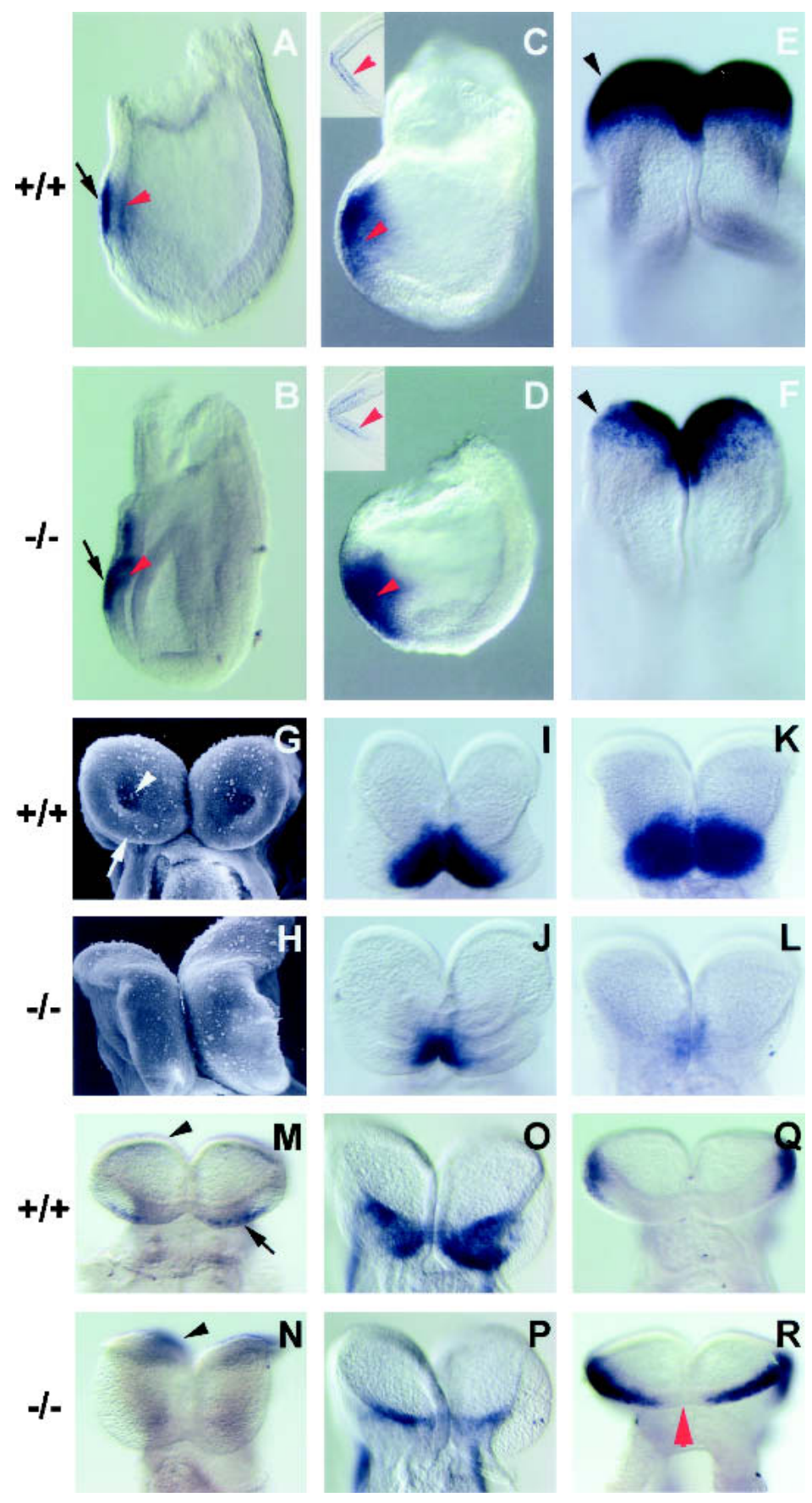

Figure 4. The patterning of the anterior visceral endoderm (AVE) and anterior neuroectoderm during early development is normal in Six3-null embryos; lack of rostral forebrain is detected at the early (4-6) somite stage. $(A)$ Whole-mount in situ hybridization of $\sim$ E7.0-E7.5 wildtype embryos showed strong expression of Hesx 1 in the anterior endoderm (black arrow) and weak expression in the overlying epiblast (anterior ectoderm) from which the future forebrain will develop (red arrowhead). (B) A similar pattern of Hesx1 expression was observed in Six3-mutant littermates. $(C)$ At the neural-plate stage (E7.5-E8.0), Hesx 1 expression was more abundant in the anterior neuroectoderm (future forebrain region) of the wild-type epiblast (red arrowhead). (D) No obvious alterations were detected in the mutant littermates. (E) At the 1-2-somite stage, Hesx 1 expression was observed in the most anterior neuroectoderm of wild-type embryos (black arrowhead) including the floor of the diencephalic portion of the primitive forebrain. $(F)$ A reduction in the level of Hesx1 expression was initially detected in the margins of the anterior neuroectoderm of Six3-null littermates. $(G)$ Scanning electron micrographs of E8.5 embryos at the 5-8-somite stage showed that the optic pit evaginations (white arrowhead) and the anterior neural ridge (white arrow) evident in the wild-type embryos were absent in Six3-null littermates $(H)$, as a result of the lack of rostral forebrain; the midbrain region appeared to be anteriorly expanded. At the 4-6-somite stage $(I)$, Hesx 1 was widely expressed in the rostral region of the anterior neuroectoderm (future forebrain) of wild-type embryos; in Six3-null littermates (J), Hesx1 expression was restricted to a narrow medial domain in the most anterior part of the mutant neural plate. $(K) R_{x}$ was expressed in the anterior neuroectoderm of wild-type embryos but was barely detectable in the Six3-null littermates $(L)$. Fgf8 was expressed in the anterior neural ridge (ANR; arrow) and isthmus (arrowhead) of wild-type embryos $(M)$; Fgf8 was not detected in the rostral neuroectoderm (ANR is missing) but appeared unaffected in the isthmus (arrowhead) of the Six3-null embryos $(N)$. (O) Bf1 was expressed in the anterior neural plate and underlying non-neural ectoderm of wild-type embryos. $(P) B f 1$ expression remained only in the nonneural ectoderm of Six3-null littermates. (Q) During the early (3-5) somite stage, Pax3 expression demarcated the prospective alar midbrain region in wildtype embryos. In Six3-null littermates $(R), P a x 3$ expression had already extended beyond its normal midbrain boundary into the anterior part of the neuroectoderm, suggesting that in the mutant embryos this region has adopted a midbrain identity; Pax3 remains negative in the most paramedial region (red arrowhead), an area in which Hesx1 expression $(J)$ was still detected. littermates (Fig. 5C). At this early somite stage, no differences in the expression of the midbrain marker Pax2 were observed (data not shown). Despite the differences in the levels of expression of Wnt1, no obvious morphologic alterations were detected in Six3-heterozygous mice. A few hours later (6-8-somite stage), ectopic anterior expansion of Wnt1 expression (Fig. 5E, arrowheads) was evident in the mutant embryos in the region rostral to its normal midbrain expression boundary (arrow). These data suggest that Six3 function is required to specifically repress Wnt1 expression from the anterior neu- roectoderm during early (headfold to early somite stage) embryonic development.

To test this possibility, we performed electroporation of a full-length Six3 expression construct in HamburgerHamilton $(\mathrm{HH})$ stage 5 chick embryos that were then allowed to develop until HH stage 8-9. As shown in Figure $5 \mathrm{~F}$ and $\mathrm{G}$, normal Wnt1 mRNA expression was detected in the unelectroporated left side of the CNS but not in the electroporated contralateral side (Fig. 5G, arrowheads); coinjections of green fluorescent protein were used to monitor the electroporated side (Fig. 5F). No al- 

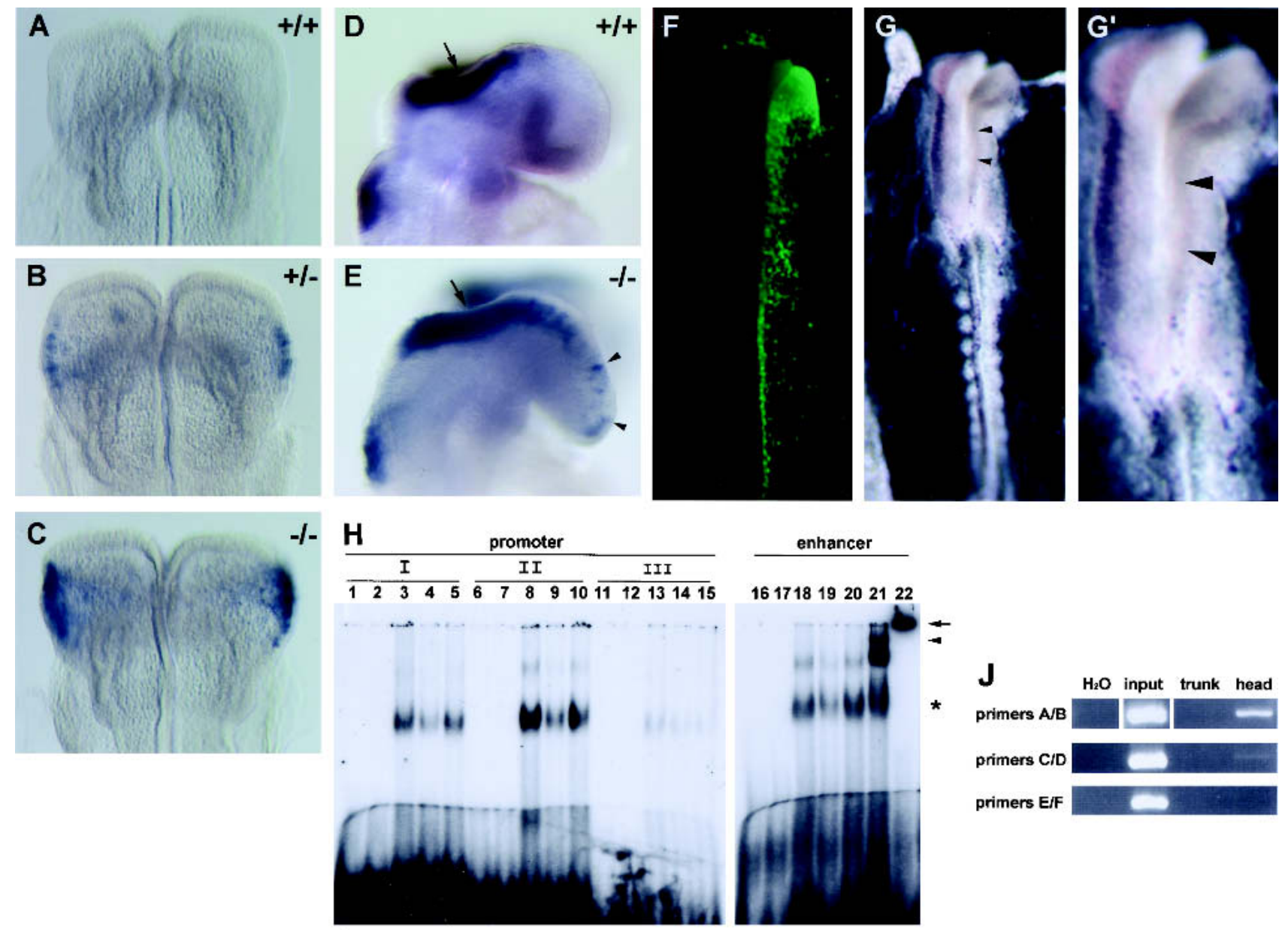

I

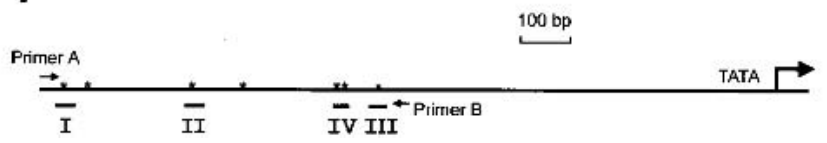

Figure 5. Six 3 represses $W n t 1$ gene activity during vertebrate head development. (A) At the early (1-2) somite stage, Wnt1 expression was almost undetectable in wild-type embryos. $(B)$ Weak expression of Wnt1 was detected in the developing midbrain of the Six3heterozygous littermates. $(C)$ The level of Wnt1 expression was the highest in the comparable region of the Six3-null littermates. (D) A few hours later (6-8-somite stage), normal Wnt1 expression demarcated the developing midbrain (arrow). (E) In the Six3-null littermates, Wnt1 expression in the midbrain territory (arrow) was maintained, and the ectopic rostral expansion of its expression was quite evident at this stage anterior to the midbrain territory (arrowheads). (F) Green fluorescent protein was expressed throughout the electroporated right side of the CNS of HH stage 8-9 chicken embryos. (G) Whole-mount in situ hybridization of Six3-electroporated embryos showed normal Wnt1 expression along the CNS on the nonelectroporated left side; no Wnt1 expression was observed on the Six3-electroporated contralateral side (arrowheads). $\left(G^{\prime}\right)$ A magnification of the electroporated embryo shown in $G$. (H) EMSA assay shows that bacteria-expressed GST-Six3 fusion protein binds to the Wnt1 promoter elements I and II (lanes 3,8) strongly, very weakly to element III (lane 13), and not at all to element IV (data not shown). No specific binding was detected when using as negative controls either probes I, II, and III alone (lanes 1,6,11), or probes I, II, and III together with GST protein (lanes 2,7,12). The binding of Six3 to probes I and II can be competed when using 400× molar excess of their corresponding wild-type unlabeled oligonucleotides (lanes 4,9), but not when using similar amounts of unlabeled mutated oligonucleotides (lanes 5,10). (Lane 18) The specific binding of the GST-Six3 fusion protein to the 40-bp Wnt1 3'-enhancer element. This element alone (lane 16) or together with GST protein (lane 17) does not show any specific binding. GST-Six 3 fusion protein bound to this Wnt1 enhancer element can be competed when using 100× molar excess of unlabeled wild-type oligonucleotide (lane 19), but not when using a mutated form (lane 20). The binding complex is supershifted by either an anti-Six3 antibody or anti-GST antibody (lanes 21,22). ${ }^{\star}$, the binding complex of GST-Six3 and DNA; arrowhead, supershifted binding complex using anti-Six3 antibody; arrow, supershifted binding complex using anti-GST antibody. (I) An 700-bp DNA fragment located 5' of the Wnt1 transcriptional initiation site includes seven clustered regions containing putative Six3 DNA-binding motifs $\left({ }^{*}\right)$. Primers A and B were used for the ChiP assay. ( $J$ ) ChiP assay on E8.5 dissected head and trunk regions of wild-type embryos showing in vivo recruitment of Six3 to the Wnt1 5'-promoter (primers A/B) and 3'-enhancer (primers C/D) regions. No recruitment of Six3 was detected when using primers (primers E/F) against an unrelated $5^{\prime}$ genomic region of Wnt1. Specific PCR amplification was only observed when using DNA extracted from the head region. 
terations in the normal expression of other midbrain markers such as Pax3 or En2 were observed after electroporation of Six3 (data not shown). This result indicated that Six 3 could repress Wnt 1 expression in vivo.

We previously demonstrated that mouse Six3 is a potent transcriptional repressor that interacts with Grouchorelated protein members, and we identified a typical consensus core ATTA motif as the Six3 DNA-binding motif (Zhu et al. 2002). A conserved 110-bp regulatory sequence within the $3^{\prime}$ Wnt 1 enhancer contributes to the correct spatial expression of this gene in the developing nervous system (Echelard et al. 1994; Iler et al. 1995; Rowitch et al. 1998). Within this fragment, an identified A/T-rich consensus homeodomain-binding site was proposed to be required to repress Wnt1 expression in the developing forebrain; specific mutations of this site extended the rostral boundary of Wnt1/lacZ staining in transgenic embryos (Iler et al. 1995; Rowitch et al. 1998), a result that was reminiscent of that observed in our Six3-null embryos. A 40-bp sequence located within the 110-bp enhancer element, including the putative homeobox-binding sites (Iler et al. 1995; Rowitch et al. 1998), as well as two different 30-35-bp fragments containing multiple ATTA motifs identified by visual inspection of the region immediately $5^{\prime}$ of the Wnt 1 promoter region (Fig. 5I), were capable of binding GST-Six3 fusion protein in an electrophoretic mobility shift assay (EMSA; Fig. 5H). The different amounts of cold competitor required to shift the Six3 probe in those assays indicated that the Wnt1 $3^{\prime}$ enhancer element has a higher binding affinity for Six 3 than the Wnt1 5' promoter region. To conclusively determine whether Wnt1 is a direct target for Six3 in vivo repression, we performed chromatin immunoprecipitation (ChIP) assays using the prospective head and trunk territories of E8.5 wild-type embryos (Fig. 5J). This assay demonstrated that Six3 protein present in the embryonic head territory is bound to elements located within the 110-bp fragment of the $3^{\prime}$ Wnt1 enhancer and to A/T-rich elements upstream of the Wnt1 transcription unit; no binding was observed in the trunk region, which did not express Six3. Similar results were also obtained when using Six3-transfected p19 cells (data not shown). These results indicated that Six 3 can bind to the $W n t 1$ regulatory sequences at a variety of sites in vivo and in vitro, suggesting that Six 3 is a direct transcriptional repressor of Wnt1 during anterior head development. It should also be mentioned that cotransfection of Six3 into various cell lines repressed expression of those two different Wnt1 promoter regions; however, these results were difficult to reproduce consistently because of the very low basal activity of the Wnt1 promoter/enhancer elements in all tested cell lines.

Six3 injections rescue the zebrafish headless phenotype

Formation of the forebrain is drastically affected (Fig. 6E) in zebrafish mutants such as headless (hdl; mutation in the tcf3 gene) and masterblind (mutation in the Gsk3binding domain of the axin gene), in which Wnt pathway components are mutated (Kim et al. 2000; Heisenberg et al. 2001; van de Water et al. 2001). Several aspects of these mutant phenotypes, including the ectopic rostral expansion of Wnt signaling (Kim et al. 2000), resemble the phenotype of Six3-null mouse embryos. In addition, six3 expression is drastically reduced in headless mutant embryos (Kim et al. 2000). Repression of Wnt targets by Tcf3 may be necessary to allow the expression of genes required for forebrain development (Kim et al. 2000). At the same time, it has been previously shown that overexpression of an activated form of Six3 (VP16-Six3) in zebrafish embryos leads to eye and forebrain hypoplasia because of a reduction in the expression domains of the anterior neural markers $\mathrm{rx} 2$, pax2, and emx1 (Kobayashi et al. 2001), a result supporting the proposal that Six3 acts as a transcriptional repressor during vertebrate forebrain development. Taking these data into consideration and to further corroborate whether Six 3 can repress wnt 1 expression and therefore alter antero-posterior neural patterning in vivo, we analyzed its activity in zebrafish embryos. Injection of mouse Six3 mRNA into wild-type and hdl-mutant zebrafish embryos resulted in partial or complete repression of wnt1 expression (Fig. 6B,D; data not shown); thus, the ability of Six3 to repress wnt1 transcription is evolutionarily conserved in vertebrates. Strikingly, ectopic expression of mouse Six3 mRNA in one-cell-stage hdl embryos repressed wnt1 expression and rescued the headless phenotype, as indicated by the appearance of normal eyes (Fig. 6F). These results provide additional support for the hypothesis that Six3 promotes anterior neural fates primarily via the negative regulation of Wnt signaling. Furthermore, they also suggest that Six3 functional activity is part of a feedback regulatory loop operating in the anterior neuroectoderm that includes members of the Wnt signaling pathway. This proposal is supported by the induction of ectopic Six3 expression in the posterior CNS of chicken embryos after electroporation of Gsk3 (Fig. 7A), and by the specific repression of Six3 expression in the anterior neuroectoderm (e.g., Otx2 expression is not affected) after electroporation with a Wnt3A expression plasmid (Fig. 7B). This result indicates that, directly or indirectly, Gsk3, a negative regulator of the Wnt pathway up-regulates Six3 expression.

\section{Discussion}

In this paper we have established that in mice, the absence of Six3 activity results in telencephalic and optopreopto-hypothalamic truncations and partial caudalization of the mutant head, as indicated by the rostral extension of the pattern of expression of two dorso-caudal diencephalic markers (Wnt1 and Pax3) without apparently affecting that of the normal dorso-ventral markers (Pax6, Shh, and Nkx2.1) or the formation of the ZLI. Rostral expansion of Wnt1 expression was also previously reported in the case of Otx1 $1^{-/-}$; Otx2 $2^{+/-}$mutant mice (Acampora et al. 1997).

The detailed expression analysis performed using a variety of markers whose activity is necessary in the ante- 
Figure 6. Ectopic murine Six 3 represses wnt1 expression and rescues forebrain deficiency in hdl mutant zebrafish embryos. (A) Whole-mount in situ hybridization analysis (Marlow et al. 1998) of wnt1 expression in wild-type embryos $10 \mathrm{~h}$ postfertilization. (B) Injection of synthetic murine Six3 mRNA into wild-type embryos at the one-cell stage (Thisse and Thisse 1998) repressed endogenous wnt 1 expression in $90 \%$ of the embryos (n = 164). (C) wnt1 (arrowhead) and six3 (arrow) expression was observed in control (uninjected) hdl embryos. In these embryos, an enlarged wnt1 expression domain was observed in $91 \%$ of cases $(\mathrm{n}=45)$. (D) Mouse Six 3 was overexpressed in hdl embryos as described (Thisse and Thisse 1998; van de Water et al. 2001); $2 \%$ of the injected embryos maintained an enlarged Wnt 1 expression domain, whereas the remaining $98 \%$ exhibited normal, reduced, or absent expression $(\mathrm{n}=63)$. (E) The lack of eyes normally seen in control hdl mutants $2 \mathrm{~d}$ postfertilization was suppressed in a mutant sibling injected with mouse Six3 mRNA $(F)$. Of the control hdl embryos $(\mathrm{n}=153), 38 \%$ lacked eyes and $62 \%$ exhibited small eyes. In contrast, $90 \%$ of the mutant embryos that received mouse Six3 mRNA injections $(\mathrm{n}=89)$ had normal eyes, $5 \%$ had small eyes, and $5 \%$ had abnormal morphology.
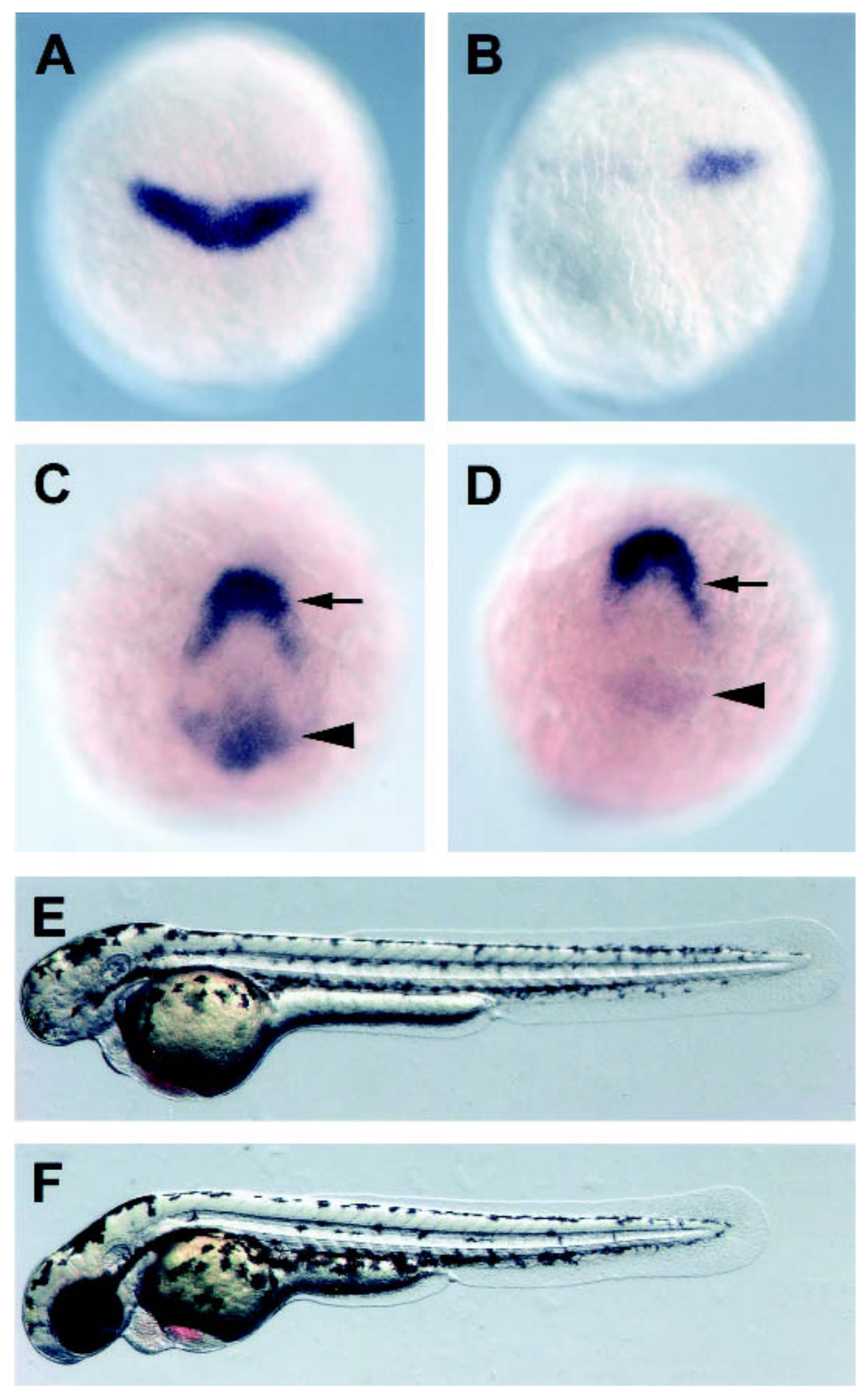

rior neural plate during vertebrate forebrain development allowed us to demonstrate that although anterior neural induction occurs in Six3-null embryos, the subsequent inductive steps leading to rostral forebrain formation are arrested. We concluded that normal forebrain development and regional antero-posterior head specification requires Six3 activity in the anterior neuroectoderm during the period between the headfold and early somite stages (E7.5-E8.0).

We propose that in Six3-null embryos anterior neural induction occurs. However, the ectopic rostral expansion of Wnt1 expression overrides the molecular program normally required for rostral forebrain formation. Thus, rostral forebrain formation is never initiated, and the caudal diencephalon territory abnormally expands into the anterior region of the mutant head. These results suggest that during specification of the different brain regions, Six3 participates in the specific repression of Wnt1 expression from the anterior neuroectoderm during early (headfold to early somite stage) embryonic development. In addition, Six3 activity in the anterior neuroectoderm could also be required for the induction and/or maintenance of the ANR.

In zebrafish, forebrain patterning is controlled, at least in part, by the expression of Tlc, a secreted Wnt inhibitor (Houart et al. 2002); therefore, it is possible that Six3 also operates in this pathway by inducing the expression of this inhibitor. It could be argued that loss of Tlc expression, and probably of other not yet identified Wnt antagonists expressed normally in the anterior neuroectoderm, also leads to increased Wnt1 signaling in headless mutant fish. Notably, Houart at al. (2002) demonstrated that Tlc can lead to repression of an enlarged wnt1 expression domain in embryos lacking Anterior Neural 


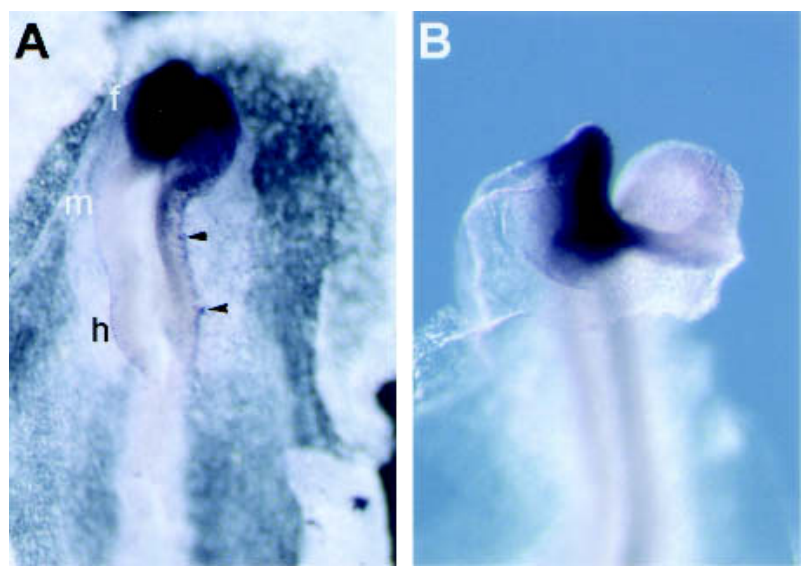

Figure 7. (A) A Gsk3 expression vector was electroporated on one side of the midbrain $(\mathrm{m})$ and hindbrain $(\mathrm{h})$ regions of $\mathrm{HH}$ stage 5 chicken embryos. In situ hybridization of $\mathrm{HH}$ stage $8-9$ embryos revealed ectopic Six3 activation throughout the electroporated side of the midbrain and hindbrain regions (arrowheads); normal Six3 expression was seen in the forebrain (f) region. (B) Wnt3A electroporation repressed Six3 expression in one side of the anterior neuroectoderm.

Border. As Tlc is an extracellular protein, the mechanism through which wnt1 expression is regulated was not clear. An excess of Wnt signaling (Tlc-depleted, headless mutants), and thus reduced GSK-3 activity, can lead to reduced six 3 expression as indicated by our chick electroporation results, showing that, directly or indirectly, GSK-3 can induce Six3 expression. Therefore, our work provides the first molecular mechanism through which excess Wnt signaling in the anterior neuroectoderm can impact Wnt1 gene transcription.

It has been previously proposed that Six 3 function could be necessary for forebrain formation (Oliver et al. 1995; Kobayashi et al. 2001). Here we provide direct genetic evidence showing that, indeed, Six 3 activity in the anterior neuroectoderm is required during the headfold to early somite stage (E7.5-E8.0) for the development of the mammalian rostral forebrain.

With the exception of Hesx1, rostral head truncations generated previously by gene inactivation were caused by defects in the AVE (Thomas and Beddington 1996; Shawlot et al. 1999; Martinez-Barbera and Beddington 2001; Perea-Gomez et al. 2001), node (Bachiller et al. 2000), or axial mesendoderm (Mukhopadhyay et al. 2001). Activity of the homeobox gene Hesx 1 is required in the anterior neural ectoderm, and variable forebrain truncations have been observed in Hesx1-null embryos (Martinez-Barbera et al. 2000; Martinez-Barbera and Beddington 2001). Therefore, Six3 and Hesx 1 are among the earliest genes functioning in the anterior neural plate during head patterning.

A great deal of evidence supports the hypothesis that the level of Wnt activity specifies different posterior-toanterior fates within the neural plate (Niehrs 1999; Heisenberg et al. 2001; Kiecker and Niehrs 2001; Houart et al. 2002). In this model, suppression of Wnt signaling in the paraxial mesoderm during gastrulation (Nordstrom et al. 2002) and subsequently within the anterior neuroectoderm is required for the formation of anterior neural structures (i.e., the rostral forebrain; Houart et al. 2002). Our results not only provide support for this hypothesis, but also demonstrate that during normal forebrain development, Six3 directly represses Wnt1 expression in the anterior vertebrate neuroectoderm fated to become forebrain.

Although no alterations in anterior neural induction were observed at the neural-plate stage, removal of Six3 activity from the anterior neuroectoderm at around the $1-2$-somite stage resulted in the premature, concentration-dependent induction of Wnt1 expression in the putative midbrain region. A few hours later, an abnormal ectopic anterior extension of the Wnt1 expression domain was evident in the Six 3 mutant head; this rostral expansion of Wnt1 expression overrode the rostral forebrain-inducing process, thereby resulting in an expanded caudal diencephalic region. In addition, our in vivo binding assays revealed that Six 3 represses Wnt 1 expression by binding to its $3^{\prime}$ enhancer and to elements located within its $5^{\prime}$ promoter region. These experiments not only identified Six3 as a key player in vertebrate head development, but also demonstrated the existence of another regulatory step in the complex Wnt signaling pathway, the direct repression of Wnt 1 expression by a transcription factor in the mammalian anterior neural plate at the late headfold-early somite stage, a step that is probably required for the maintenance of the anterior neural fates. This Six3-promoted Wnt1-free anterior territory appears to be a prerequisite for the subsequent establishment of the anterior signaling center, which, in turn, induces the expression of Fgf8 and other downstream genes (e.g., $B f 1$ and $R x$ ) participating in the further expansion and maturation of the forebrain (Shimamura and Rubenstein 1997). Our results also suggest that this process is probably part of a cross-regulatory loop and provides the first molecular mechanism through which excess Wnt signaling in the anterior neuroectoderm can impact Wnt1 gene transcription. Thus, Six3 is an essential regulator of vertebrate forebrain development.

\section{Materials and methods}

\section{Functional inactivation of Six3}

$\mathrm{Six}^{-/-}$mice were generated by an in-frame fusion of a bluntended 5.1-kb SmaI-XhoI fragment containing LacZpA-pGKNeopA sequences into the NcoI blunt-ended XhoI site that was 22 amino acids downstream of the first initiation methionine. The XhoI site was lost during the cloning of the $3^{\prime}$ arm, and a HindIII site was inserted. W9.5 embryonic stem (ES) cells were electroporated and selected by standard procedures. Positive clones were used to generate chimeras by blastocyst injection. Southern blot analysis and PCR amplification of genomic DNA were used to identify the mutated allele.

Embryo histology and in situ hybridization analysis

Embryos were fixed $40 \mathrm{~min}$ to $1 \mathrm{~h}$ in $4 \%$ paraformaldehyde and processed as described for whole-mount in situ hybridization 
(Belo et al. 1997). Cartilage and bones were stained with alcian blue and alizarin red.

\section{Chicken electroporation}

Electroporation of HH stage 5 chicken embryos was done in vitro, as previously described (Kobayashi et al. 2002).

\section{Electrophoretic mobility shift assay (EMSA)}

Pure GST and GST-Six3 fusion proteins were prepared for the EMSA as previously described (Zhu et al. 2002). To perform this assay, we used the synthetic sense (GCCTGTATTTATTACT CTCCCATTGTCACTAATTGAGGTAATTAT) and antisense oligonucleotides spanning the sequence of the mouse $3^{\prime}$ Wnt1 enhancer containing the previously identified homeodomain core sites (Iler et al. 1995; Rowitch et al. 1998) and four sets of different sense and antisense oligonucleotide pairs spanning part of the A/T-rich region $5^{\prime}$ of the Wnt1 transcriptional unit (Fig. 5; sense: I, GGCGGAATAGGCCTGTAATCCCAGCAGT CACTGGA; II, GACTAGCACATCTAATGATAAGCACAGG TTGA; III, GTACACTTTGACTAATCTCACGGGTGA; IV, GAGCCAAATTACACAATTATTTGG). Sense oligonucleotides were annealed with their corresponding antisense partner. Klenow enzyme was used to end-label the annealed sequences with $\left[\alpha{ }^{-32} \mathrm{P}\right] \mathrm{dCTP}$. The labeled probes were incubated with pure GST or GST-Six3 fusion proteins in binding buffer $25 \mathrm{mM}$ HEPES at $\mathrm{pH} 7.5 ; 100 \mathrm{mM} \mathrm{KCl} ; 1 \mathrm{mM}$ EDTA; $10 \mathrm{mM} \mathrm{MgCl}_{2}$; $0.1 \%$ NP-40; $5 \%$ glycerol; and 1 mM DTT) supplemented with $0.6 \mu \mathrm{g} / \mu \mathrm{L}$ poly $(\mathrm{dI}-\mathrm{dC})$. Competition of the specific protein-DNA complexes was performed with $100 \mathrm{M}$ excess (for the $3^{\prime}$ enhancer) or $400 \mathrm{M}$ excess (for the $5^{\prime}$ regulatory region) of either unlabeled wild-type or mutated oligonucleotides. Wild-type Wnt1 3'-enhancer oligonucleotide was mutated at all three putative core homeodomain protein-recognition sequences (Iler et al. 1995; Rowitch et al. 1998): ATTA was mutated to AGCA, TAAT to TGCT, and TAATTA to TAAGCA. Wild-type TAAT core present in $W n t 15^{\prime}$ oligonucleotides was mutated to TGCT. For supershift of the protein-DNA complexes, rabbit antimouse Six 3 antibody $(0.5 \mu \mathrm{L})$ or goat anti-GST antibody $(0.5 \mu \mathrm{L}$; Amersham Pharmacia Biotech) was added to the binding mix. The DNA-protein complex was resolved in 5' nondenaturing protein gel and visualized by autoradiography.

\section{ChIP assay}

For the in vivo ChIP experiments, extracts were prepared from 21 E8.5 (3-6 somites) wild-type mouse embryonic heads and trunks. Embryos were microdissected in high-glucose DMEM supplemented with $10 \%$ Fetal Calf Serum. Heads and trunks were washed twice in PBS and treated for 3 min with ES cellgrade trypsin-EDTA. Following gentle pipetting, tissue was cross-linked with $1 \%$ formaldehyde at $37^{\circ} \mathrm{C}$ for $10 \mathrm{~min}$. Chromatin extraction and immunoprecipitations were performed by using a ChIP assay kit (Upstate Biotechnology) according to the manufacturer's protocol. The amount of chromatin was normalized by optic density. Protein-DNA cross-linking was reversed by overnight incubation at $65^{\circ} \mathrm{C}$. A PCR purification kit (QIAGEN) was used to recover DNA in $50 \mu \mathrm{L}$. The following PCR primers against the 5' Wnt 1 promoter region were used: primer A (5' -CTTGAGTTGGGCAGGTACGGT-3') and primer B (5'-AGGGGGAGTGTAAGCGTCGGT-3'; Fig. 5I). For the Wnt1 $3^{\prime}$-enhancer element the following PCR primers were used: primer C (5'-CGTCAGCCTGGATTAATCTTC G-3') and primer D (5'-TTGGGAGACACTTCGTGAACG-3'). As controls, primers against an unrelated region of the Wnt1 enhancer region were used: primer E $\left(5^{\prime}\right.$-GTGCGAGAGTGTG TACGCGTT- $\left.3^{\prime}\right)$ and primer $\mathrm{F}\left(5^{\prime}\right.$-CCTATCCCCTCCTTAA GCGACA-3'). Because of the difficulty of the assay, the experiment using the embryonic extracts was performed just once; however, all possible negative and positive controls were included (e.g., positive band only when using the head region but not the Six3-free trunk, no genomic DNA contamination), and, on the basis of other supporting evidence provided in this paper, we believe that the result is clear and convincing. In addition, similar results were observed when using extracts generated from p19 cells transfected with CMV-Six3 plasmids (data not shown).

\section{Acknowledgments}

We thank J. Morgan, S. Self, I. Lagutina, B. Bowling, and M. Torres for help during this project; C. Nagy, L. Emmons, and J. Raucci (all of the Transgenic Core Facility) for performing injections; D. Fakete (Scientific Imaging Shared Resources) for generating the scanning electron micrographs; A. McArthur (Scientific Editing) for editing this manuscript; G. Grosveld for very helpful advice; C. Abate-Shen, R. Di Lauro, A. Joyner, A. McMahon, D. Rowitch, G. Martin, H. Clevers, C, Niehrs, A. Simeone, P. Mathers, A. Kikuchi, R. Toyama, R. Chitnis, and S.W. Wilson for plasmids; A. Chitnis for hdl fish; and E.M. DeRobertis for valuable comments and suggestions on the manuscript. This work was supported in part by grants DGIC PB98-0397 and PI-64/00862/FS/01 to L.P.; Ministry of Education, Culture, Sports, Science and Technology of Japan grant to K.S.; Pew Scholars Program in Biomedical Sciences to L.S.-K.; and the National Institutes of Health grants EY12162 and GM58462, Cancer Center Support CA-21765, and the American Lebanese Syrian Associated Charities (ALSAC) to G.O.

The publication costs of this article were defrayed in part by payment of page charges. This article must therefore be hereby marked "advertisement" in accordance with 18 USC section 1734 solely to indicate this fact.

\section{References}

Acampora, D., Avantaggiato, V., Tuorto, F., and Simeone, A. 1997. Genetic control of brain morphogenesis through Otx gene dosage requirement. Development 124: 3639-3650.

Bachiller, D., Klingensmith, J., Kemp, C., Belo, J.A., Anderson, R.M., May, S.R., McMahon, J.A., McMahon, A.P., Harland, R.M., Rossant, J., et al. 2000. The organizer factors Chordin and Noggin are required for mouse forebrain development. Nature 403: 658-661.

Belo, J.A., Bouwmeester, T., Leyns, L., Kertesz, N., Gallo, M., Follettie, M., and De Robertis, E.M. 1997. Cerberus-like is a secreted factor with neutralizing activity expressed in the anterior primitive endoderm of the mouse gastrula. Mech. Dev. 68: 45-57.

Camus, A., Davidson, B.P., Billiards, S., Khoo, P., Rivera-Perez, J.A., Wakamiya, M., Behringer, R.R., and Tam, P.P 2000. The morphogenetic role of midline mesendoderm and ectoderm in the development of the forebrain and the midbrain of the mouse embryo. Development 127: 1799-1813.

Crossley, P.H. and Martin, G.R. 1995. The mouse Fgf8 gene encodes a family of polypeptides and is expressed in regions that direct outgrowth and patterning in the developing embryo. Development 121: 439-451.

Davis, C.A. and Joyner, A.L. 1988. Expression patterns of the homeo box-containing genes En-1 and En-2 and the proto- 
oncogene int-1 diverge during mouse development. Genes \& Dev. 2: 1736-1744.

Echelard, Y., Vassileva, G., and McMahon, A.P. 1994. Cis-acting regulatory sequences governing Wnt-1 expression in the developing mouse CNS. Development 120: 2213-2224.

Heisenberg, C.P., Houart, C., Take-Uchi, M., Rauch, G.J., Young, N., Coutinho, P., Masai, I., Caneparo, L., Concha, M.L., Geisler, R., et al. 2001. A mutation in the Gsk3-binding domain of zebrafish Masterblind/Axin1 leads to a fate transformation of telencephalon and eyes to diencephalon. Genes \& Dev. 15: 1427-1434.

Houart, C., Caneparo, L., Heisenberg, C., Barth, K., Take-Uchi, M., and Wilson, S. 2002. Establishment of the telencephalon during gastrulation by local antagonism of Wnt signaling. Neuron 35: 255-265.

Iler, N., Rowitch, D.H., Echelard, Y., McMahon, A.P., and Abate-Shen, C. 1995. A single homeodomain binding site restricts spatial expression of Wnt-1 in the developing brain. Mech. Dev. 53: 87-96.

Kiecker, C. and Niehrs, C. 2001. A morphogen grandient of $\mathrm{Wnt} / \beta$-cateninc signaling regulates anteroposterior neural patterning in Xenopus. Development 128: 4189-4201.

Kim, C.H., Oda, T., Itoh, M., Jiang, D., Artinger, K.B., Chandrasekharappa, S.C., Driever, W., and Chitnis, A.B. 2000. Repressor activity of Headless/Tcf3 is essential for vertebrate head formation. Nature 407: 913-916.

Kobayashi, M., Nishikawa, K., Suzuki, T., and Yamamoto, M. 2001. The homeobox protein Six3 interacts with the Groucho corepressor and acts as a transcriptional repressor in eye and forebrain formation. Dev. Biol. 232: 315-326.

Kobayashi, D., Kobayashi, M., Matsumoto, K., Ogura, T., Nakafuku, M., and Shimamura, K. 2002. Early subdivisions in the neural plate define distinct competence for inductive signals. Development 129: 83-93.

Lagutin, O., Zhu, C.C., Furuta, Y., Rowitch D.H., Mc.Mahon, A.P., and Oliver, G. 2001. Six3 promotes the formation of ectopic optic vesicles in mouse embryos. Dev. Dyn. 221: 342-349.

Lazzaro, D., Price, M., de Felice, M., and Di Lauro, R. 1991. The transcription factor TTF-1 is expressed at the onset of thyroid and lung morphogenesis and in restricted regions of the fetal brain. Development 113: 1093-1104.

Marlow, F., Zwartkruis, F., Malicki, J., Neuhauss, S.C., Abbas, L., Weaver, M., Driever, W., and Solnica-Krezel, L. 1998. Functional interactions of genes mediating convergent extension, knypek and trilobite, during the partitioning of the eye primordium in zebrafish. Dev. Biol. 203: 382-399.

Martinez-Barbera, J.P. and Beddington, R.S. 2001. Getting your head around Hex and Hesx1: Forebrain formation in mouse. Intl. J. Dev. Biol. 45: 327-336.

Martinez-Barbera, J.P., Rodriguez, T.A., and Beddington, R.S. 2000. The homeobox gene Hesx 1 is required in the anterior neural ectoderm for normal forebrain formation. Dev. Biol. 223: 422-430.

Mathers, P.H., Grinberg, A., Mahon, K.A., and Jamrich, M. 1997. The $R_{x}$ homeobox gene is essential for vertebrate eye development. Nature 387: 603-607.

Mukhopadhyay, M., Shtrom, S., Rodriguez-Esteban, C., Chen, L., Tsukui, T., Gomer, L., Dorward, D.W., Glinka, A., Grinberg, A., Huang, S.-P., et al. 2001. Dickkopf1 is required for embryonic head induction and limb morphogenesis in the mouse. Dev. Cell 1: 423-434.

Niehrs, C. 1999. Head in the WNT: The molecular nature of Spemann's head organizer. Trends Genet. 15: 314-319.

Nieuwkoop, P.D. 1952. Activation and organization of the central nervous system in amphibians. II. Differentiation and organization. J. Exp. Zool. 120: 33-81.

Nordstrom, U., Jessell, T.M., and Edlund, T. 2002. Progressive induction of caudal neural character by graded Wnt signaling. Nat. Neurosci. 5: 525-532.

Oliver, G., Mailhos, A., Wehr, R., Copeland, N.G., Jenkins, N.A., and Gruss, P. 1995. Six3, a murine homologue of the sine oculis gene, demarcates the most anterior border of the developing neural plate and is expressed during eye development. Development 121: 4045-4055.

Pasquier, L., Dubourg, C., Blayau, M., Lazaro, L., Le Marec, B., David, V., and Odent, S. 2000. A new mutation in the sixdomain of SIX3 gene causes holoprosencephaly. Eur. J. Hum. Genet. 8: 797-800.

Perea-Gomez, A., Lawson, K.A., Rhinn, M., Zakin, L., Brulet, P., Mazan, S., and Ang, S.L. 2001. Otx2 is required for visceral endoderm movement and for the restriction of posterior signals in the epiblast of the mouse embryo. Development 128: $753-765$.

Rowitch, D.H., Echelard, Y., Danielian, P.S., Gellner, K., Brenner, S., and McMahon AP. 1998. Identification of an evolutionarily conserved 110 base-pair cis-acting regulatory sequence that governs Wnt-1 expression in the murine neural plate. Development 125: 2735-2746.

Shawlot, W., Wakamiya, M., Kwan, K.M., Kania, A., Jessel, T.M., and Behringer, R. 1999. Lim1 is required in both primitive streak-derived tissues and visceral endoderm for head formation in the mouse. Development 126: 4925-4932.

Shimamura, K. and Rubenstein, J.L. 1997. Inductive interactions direct early regionalization of the mouse forebrain. $D e-$ velopment 124: 2709-2718.

Simeone, A., Acampora, D., Gulisano, M., Stornaiuolo, A., and Boncinelli, E. 1992. Nested expression domains of four homeobox genes in developing rostral brain. Nature 358: 687690.

Simeone, A., Acampora, D., Mallamaci, A., Stornaiuolo, A., D'Apice, M.R., Nigro, V., and Boncinelli, E. 1993. A vertebrate gene related to orthodenticle contains a homeodomain of the bicoid class and demarcates anterior neuroectoderm in the gastrulating mouse embryo. EMBO J. 12: 2735-2747.

Tao, W. and Lai, E. 1992. Telencephalon-restricted expression of BF-1, a new member of the HNF-3/fork head gene family, in the developing rat brain. Neuron 8: 957-966.

Thisse, C. and Thisse, B. 1998. High resolution whole-mount in situ hybridization. Zebrafish Sci. Mon. 5: 8-9.

Thomas, P. and Beddington, R. 1996. Anterior primitive endoderm may be responsible for patterning the anterior neural plate in the mouse embryo. Curr. Biol. 6: 1487-1496.

van de Water, S., van de Wetering, M., Joore, J., Esseling, J., Bink, R., Clevers, H., and Zivkovic, D. 2001. Ectopic Wnt signal determines the eyeless phenotype of zebrafish masterblind mutant. Development 128: 3877-3888.

Wallis, D.E., Roessler, E., Hehr, U., Nanni, L., Wiltshire, T., Richieri-Costa, A., Gillessen-Kaesbach, G., Zackai, E.H., Rommens, J., and Muenke, M. 1999. Mutations in the homeodomain of the human SIX3 gene cause holoprosencephaly. Nat. Genet. 22: 196-198.

Walther, C. and Gruss, P. 1991. Pax-6, a murine paired box gene, is expressed in the developing CNS. Development 113: $1435-1449$

Zhu, C.C., Dyer, M.A., Uchikawa, M., Kondoh, H., Lagutin, O., and Oliver, G. 2002. Six3-mediated auto repression and eye development requires its interaction with the Groucho family of corepressors. Development 129: 2835-2849. 


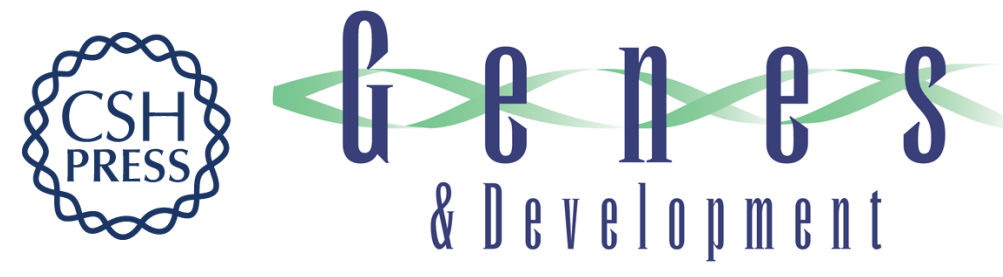

\section{Six3 repression of Wnt signaling in the anterior neuroectoderm is essential for vertebrate forebrain development}

Oleg V. Lagutin, Changqi C. Zhu, Daisuke Kobayashi, et al.

Genes Dev. 2003, 17:

Access the most recent version at doi:10.1101/gad.1059403

References This article cites 38 articles, 17 of which can be accessed free at: http://genesdev.cshlp.org/content/17/3/368.full.htmI\#ref-list-1

License

Email Alerting

Receive free email alerts when new articles cite this article - sign up in the box at the top Service right corner of the article or click here.

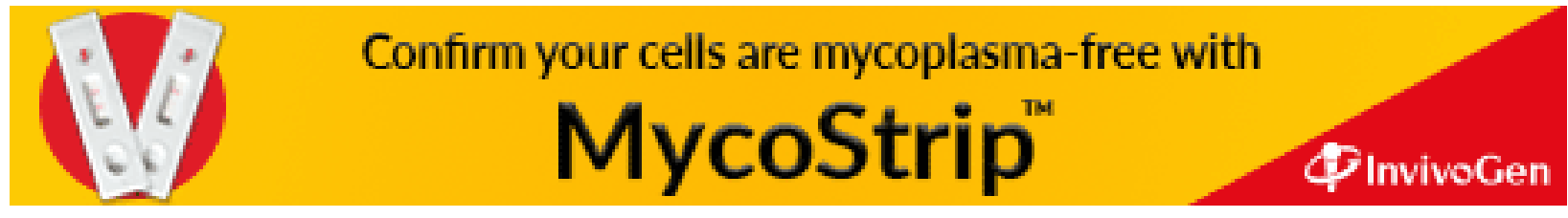

